\title{
Modeling and Validation of Gripper Induced Membrane Forces in Finite Element Forming Simulation of Continuously Reinforced Composites
}

\author{
Christian Poppe $^{1, \mathrm{a}}$, Tobias Joppich ${ }^{2, \mathrm{~b}}$, Dominik Dörr ${ }^{1, \mathrm{c}}$, Luise Kärger ${ }^{1}$, Frank \\ Henning ${ }^{1,2}$
}

${ }^{1}$ Karlsruhe Institute of Technology, Institute for Vehicle System Technology, Chair of Light-Weight Technology, Rintheimer-Querallee 2, 76131 Karlsruhe, Germany

${ }^{2}$ Fraunhofer Institute for Chemical Technology, Polymer Engineering Department, Joseph-von-Fraunhofer-Str. 7, 76327 Pfinztal, Germany

a) Corresponding author: christian.poppe@ kit.edu

b) author: tobias.joppich@ict.fraunhofer.de

c) author: dominik.p.doerr@kit.edu

\begin{abstract}
Thermoforming of multilayered, thermoplastic tape-laminates into lightweight structural vehicle components has become a considerably important process during the past years due to its large-scale production potential. However, depending on process conditions and material behavior, macroscopic defects such as fiber fracture, gapping or wrinkling are feasible. To counteract such defects, blank holders or grippers, which introduce membrane forces in the laminate, can be employed in the forming process. Usually, the number and location of grippers, as well as direction and magnitude of gripping forces are determined by a cost and time consuming "trial and error" process design. Therefore, an advanced gripper system for online monitoring of gripper forces, elongations and rotations during thermoforming is presented in this work. Along with the kinematics of the grippers, the measured forces supply the beforehand mostly unknown boundary conditions for FE forming simulation. Based on a modeling approach for FE forming simulation of CFRPs implemented in the commercially available FE solver Abaqus, appropriate modeling techniques for gripper-assisted forming are outlined. These modeling techniques are applied to two different generic geometries and the simulation results are compared with a good agreement to experimental tests.
\end{abstract}

\section{INTRODUCTION}

Forming of two-dimensional pre-products into double-curved geometries is one of the most determining process steps in manufacturing of continuously fiber-reinforced plastics (CFRP). Especially thermoforming of thermoplastic pre-impregnated, unidirectional-reinforced (UD) tapes is currently of great interest for the automotive industry due to low cycle times and recyclability [1,2]. However, thermoforming processes are influenced by several variables like temperature, layup sequence or material behavior. Dependent on these variables, manufacturing defects like e.g. fiber fracture, gapping or wrinkling are feasible. To counteract such defects, blank holders or grippers, that introduce membrane forces in the laminate, can be employed in forming processes.

By means of Finite Element (FE) forming simulation, manufacturing defects can be predicted by considering material behavior and process conditions through boundary conditions. Hence, FE forming simulation facilitates the initial validation of a process and the determination of the therefor-required process parameters. However, boundary conditions for FE forming simulation have to be parameterized and the applied modeling approaches for FE forming simulation have to be validated, to achieve meaningful results. 
In this work, an advanced gripper system, based on the work by Joppich et al. [3], for online monitoring of gripper force, translation and rotation during thermoforming is presented. Along with the kinematics of the grippers, the measured forces provide the hitherto mostly unknown boundary (load) conditions for FE forming simulation. Based on the modeling approach for FE forming simulation of CFRPs presented by Dörr et al [4,5], appropriate modeling techniques for gripper-assisted forming are outlined. These modeling techniques are applied to two different generic geometries and compared to experimental tests for the validation of FE forming simulation.

In the following, initially the applied material and process route, as well as the advanced gripper system are presented. Subsequently, suitable modeling techniques for gripper-assisted forming, containing approaches for a guided load application by means of tight or frictional fit are presented. A suitable approach for modeling process conditions is finally applied to two different generic geometries for the validation of gripper-assisted FE forming simulation. For geometry 1, boundary conditions are identified and validation is based on the comparison of translation and rotation of the grippers, while modeling the kinematics of the gripper. For geometry 2 , the simulation setup is applied unchanged, leading to meaningful validation results by demonstrating the transferability of the developed modelling approaches to a different geometry and gripper setup including two additional grippers.

\section{MATERIAL AND PROCESS}

The investigated material of this study is a carbon-fiber reinforced UD-tape with a PA6 matrix. For manufacturing of pre-consolidated blanks, which are the pre-product for thermoforming processes, spot-welded blanks are heated in an oven well above the melting temperature of the thermoplastic and are consolidated by cooling the blanks under constant transversal pressure in a hydraulic press. For this study, two different layups comprising eight single layers are investigated. The examined layups namely are a biaxial $[0 ; 90]_{2 s}$ layup and a quasiisotropic $[0 ; 45 ; 90 ;-45]_{s}$ layup.

Thermoforming processes consist of three main steps, as illustrated in Figure 1a. Initially, the laminate is gripped and precisely held within a clamping frame, followed by an automated transfer towards an infrared (IR) heating zone. After heating the laminate well above melting temperature of the thermoplastic $\left(>270{ }^{\circ} \mathrm{C}\right)$, the laminate is transferred into a hydraulic press (cf. figure 1(b)). After positioning of the laminate by means of the clamping frame, the laminate is formed by enforcing the tool stroke.

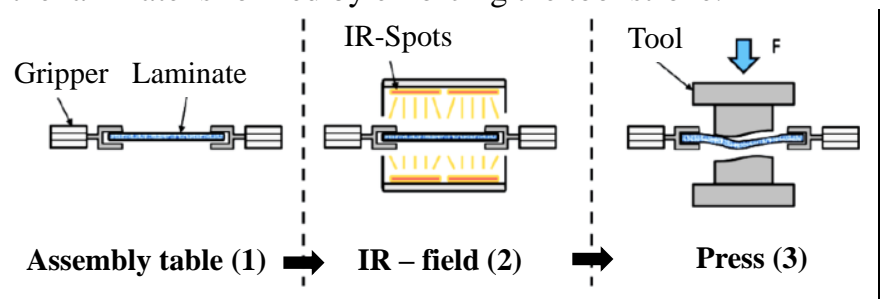

(a)

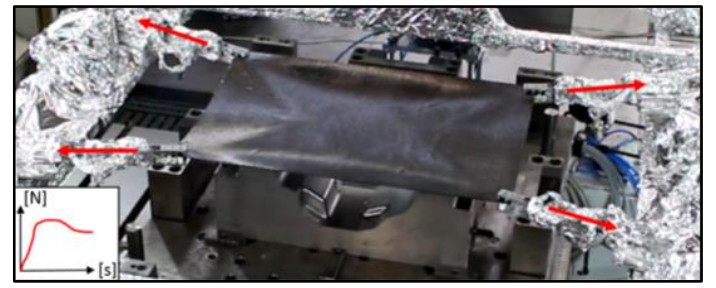

(b)

Figure 1. Schematic illustration of the thermoforming process consisting of three main process steps (a); picture of the gripper assisted forming setup during experiments (b).

\section{Gripper enhancement and process monitoring}

In order to optimize the forming process and to supply boundary conditions for FE-forming simulations, as well as to supply suitable information for the validation of FE forming simulation, process conditions have to be measured during forming. Therefor a pneumatically controlled gripper system is equipped with state of the art sensors (cf. Figure 2 ), facilitating the online monitoring of tensile force, translation and rotation of each gripper trough all process stages. By an additional rotary degree of freedom around the vertical axis of the

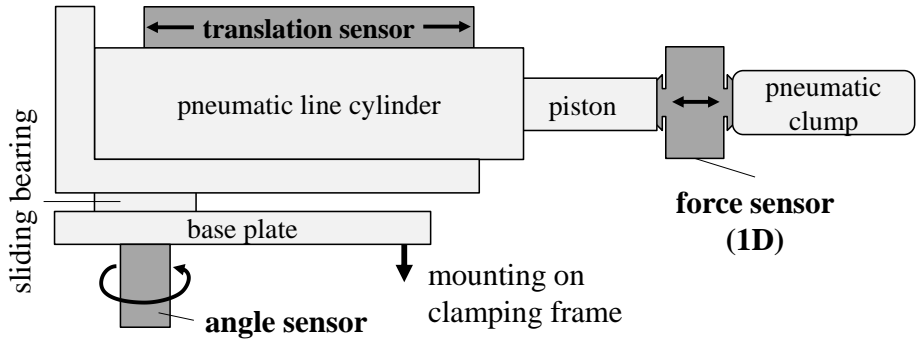

Figure 2. Schematic visualization of one enhanced gripper including the implemented sensors. 
gripper, the alignment in force direction is guaranteed. The data transfer is implemented via a WLAN-capable analog-digital converter, located at the clamping frame.

\section{FINITE ELEMENT FORMING SIMULATION}

In this study, FE forming simulations based on an approach for thermoplastic CFRPs as presented by Dörr et al. $[4,5]$ are used, which are implemented in several user subroutines of the commercially available FE solver Abaqus. In general, FE forming simulation is based on constitutive modeling of the relevant deformation mechanisms, which are usually categorized in intra-ply and interface mechanisms. To account for interface mechanisms, which are the mechanisms between the single plies of the stacked laminate, as well as between the tool and the laminate, each ply is modeled by separate element layers (cf. Figure 3(a)). The separate element-layers of each single ply are capable of modeling membrane and bending behavior (intra-ply mechanisms) in a decoupled fashion. This proceeding is crucial for FE forming simulation of CFRPs, as conventional plate theories are no longer applicable to FE forming simulation due to the very high membrane stiffness in fiber direction and the very low bending stiffness. This material characteristic can be attributed to the possible relative motion between the fibers. Furthermore, membrane and bending behavior are modeled according to a Voigt-Kelvin approach, accounting for the distinct ratedependency of the thermoplastic UD-tapes at process conditions. Beyond that, tools are modeled as rigid surfaces, as the stiffness of the tools is several orders of magnitudes higher than the stiffness of the laminate.

\section{Modelling approach for membrane force application}

To account for grippers with differing designs, modular approaches for modelling the load application by grippers or blank holders are desirable for FE forming simulation. Regarding FE modeling, gripper systems can be classified into the type of load application (tight fit, frictional fit) and the kinematics of the gripper system, in general. Modeling of a frictional fit is more likely to be used for textile draping and is modeled by according rigid surfaces under transversal pressure, inducing frictional forces into the laminate. On the contrary, modelling of a tight-fit is enabled by the application of node-to-node constraints, reducing the model of the load application area to a single reference point (cf. Figure 3(b)). The latter is used in this study due to the material behavior in the load application area, as described in the next section. To apply this approach to a multilayer laminate, the load application area is initially reduced to a reference point for each ply. Subsequently, the reference points for each ply are constrained to a single reference point, which is adopted for load application.

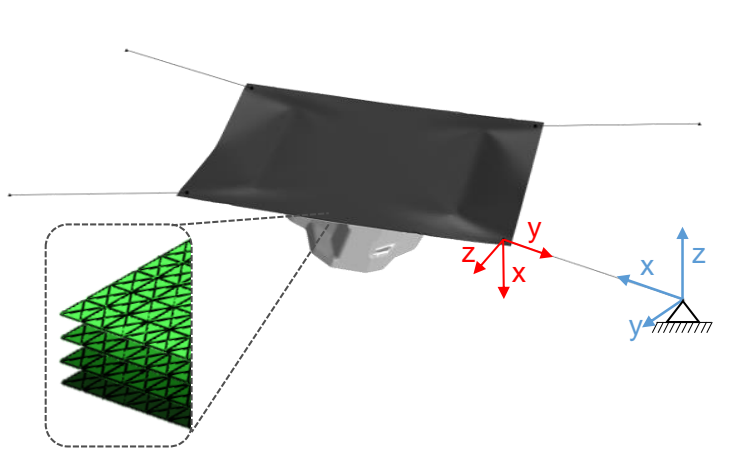

(a)

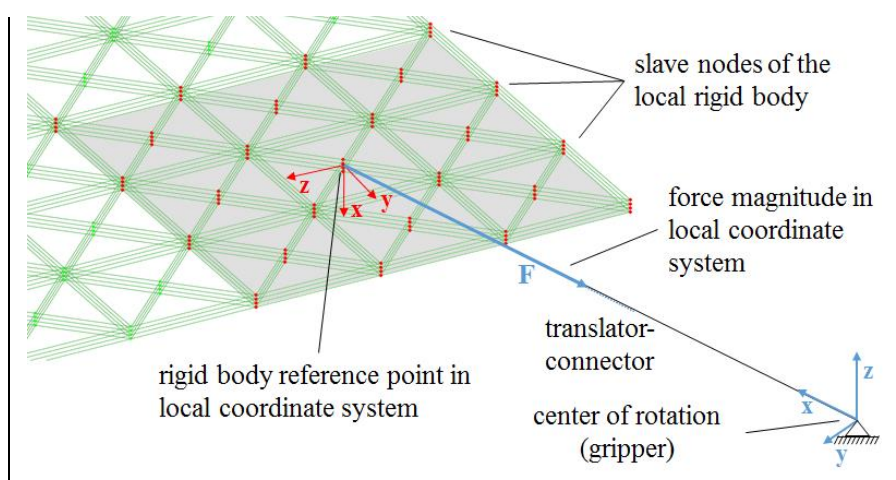

(b)

Figure 3. Visualization of the implemented simulation model in Abaqus with four element layers (a) and a detailed schematic representation of the local gripper model (b) using a rotatable rigid body in a translator defined local coordinate system.

Besides the type of load application, also the kinematics of the gripper has to be accounted for. For that purpose, so-called translator-elements are applied, which are connected on one side to the laminate reference point and on the other side to a spatially fixed point. This approach enables to model the guided translation and rotation of a gripper, by prescribing the translation and degrees of freedom at the reference points on both sides of the translator element, leading to a prober virtual representation of the experimental setup (cf. Figure 1(b)). 


\section{EXPERIMENTAL VALIDATION}

For the validation of forming simulation and the above presented modeling techniques, two complexly shaped, generic geometries comprising corner bending and beads are investigated. Geometry 1 measures a base area of 300 $\mathrm{mm} \times 416 \mathrm{~mm}$ and is manufactured with a symmetric setup comprising four grippers. On the contrary geometry 2 measures a base area of $805 \mathrm{~mm}$ x $636 \mathrm{~mm}$, which is manufactured with a symmetric setup comprising six grippers.

Grippers with a single spike mounted on a pneumatic line cylinder are applied to induce membrane forces. Due to the local shielding of the IR radiation by the gripper, a local non-melted area remains in the region of the grippers during forming. To account for this process conditions, a tight fit accompanied with a translator-element is adopted to model the load application (cf. Figure 3). The non-melted area is modeled by node-to-node rigid constraints and only the translational degrees of freedom are constrained to the vertical translator-element on both sides, to account for the spike and its possible rotation around the vertical axis. By setting the spatially fixed end of the horizontal translator element to the position on the clamping frame, the gripper kinematics is unambiguously described.

Boundary conditions are parameterized according to the measured forces on geometry 1 . It turns out, that due to friction and compression in the mechanical and pneumatic system, measured forces are identified to be 5 to $10 \mathrm{~N}$ higher than theoretically set, which is crucial information for FE forming simulation. As the measured data is adopted, a consideration of frictional and damping behavior of the gripper system is not necessary, as these mechanisms are part of the measured data.

On the one hand, simulation results are validated based on measured gripper translation and rotation for geometry 1 . On the other hand, the validated simulation setup is extended by two more grippers for the forming simulation of geometry 2 , keeping all other parameters unchanged.

\section{Geometry 1}

The results of the experimental test and the forming simulation are given in Figure 4(a) and (b) for the biaxial layup at fully closed mold. It turns out, that a good agreement of the outer contour, especially for the load application area is obtained. Beyond that, the measured translational motion and rotation of the grippers is compared to the virtual predictions in Figure 4(c), where a very good agreement between forming simulation and experimental test is observed for three of the four investigated grippers. The relative translation of gripper 4 shows a deviation between experimental test and simulation, where a measuring error cannot be excluded, as there is a good agreement for the outer contour. The presented data is collected by applying the beforehand measured forces to each gripper.

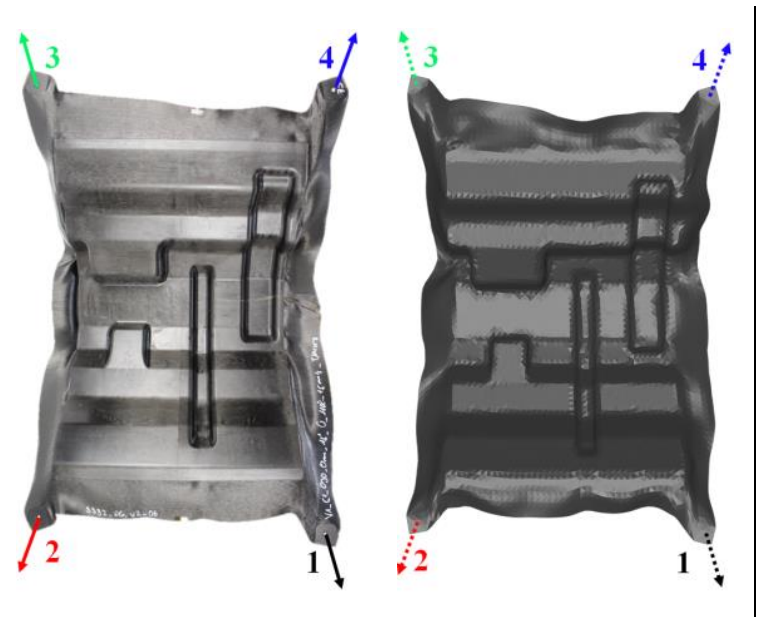

(a)

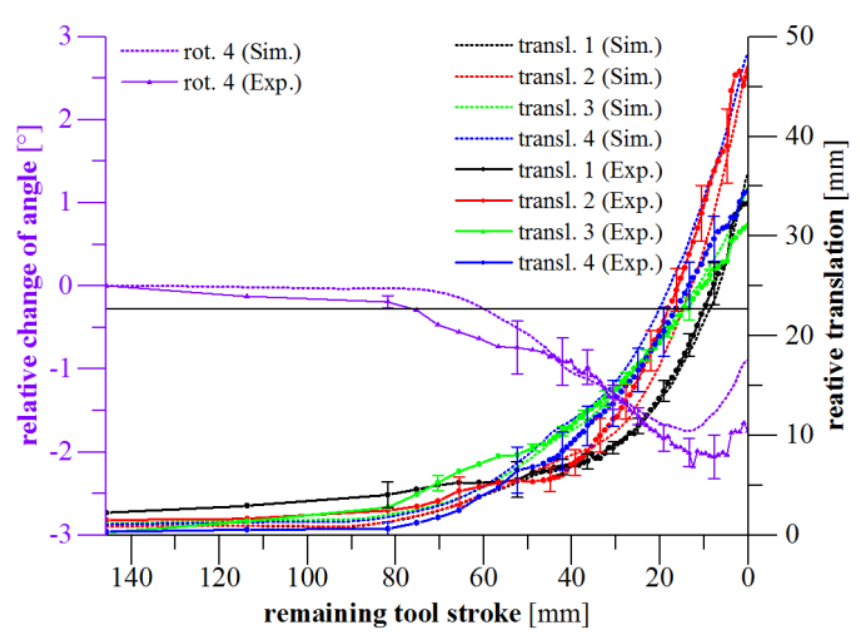

(c)

FIGURE 4. Comparison of the experimental result (a) to the simulation result (b) for a biaxial layup with 32,5 N per gripper; comparison of measured and predicted relative translations for each gripper and the rotation of gripper 4 additionally (c).

Furthermore, a tensile force variation for geometry 1 is presented in figure 5 including the relative, average translations of all four grippers (cf. Figure 5(a)) and the average change of angle of gripper 4 only (cf. Figure 5(b)), due to the limited number of input-channels of the analog-digital converter. By using three different tensile force 
levels $(7,5 \mathrm{~N}, 32,5 \mathrm{~N}$ and $80,0 \mathrm{~N})$ in both, experiment and simulations, a good validity of the modeling approach is observed for the prediction of translations of the grippers in terms of tensile force variations.

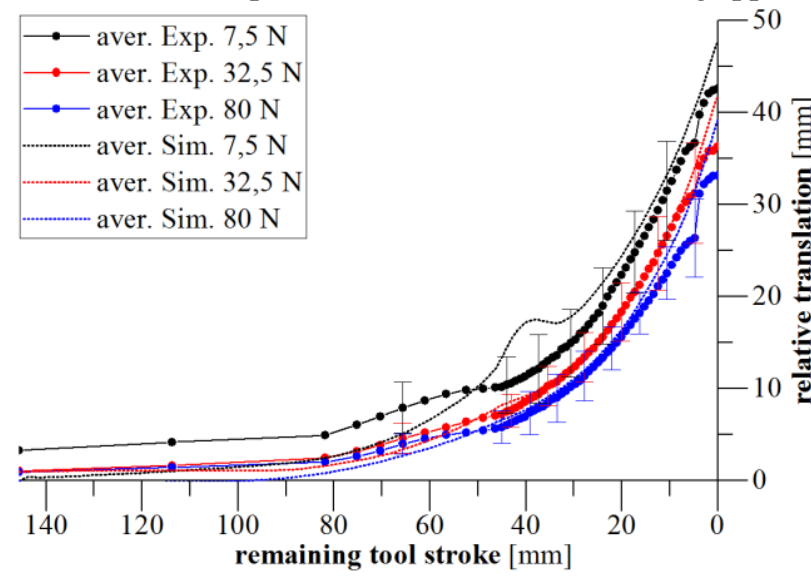

(a)

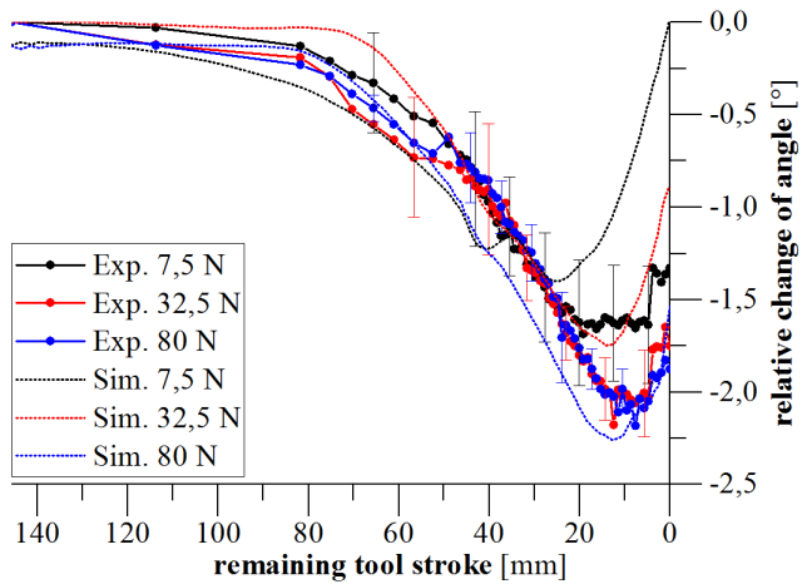

(b)

FIGURE 5. Validation for the biaxial layup and geometry 1 by means of a tensile force variation $(7,5 \mathrm{~N}-32,5 \mathrm{~N}-80 \mathrm{~N})$ during the forming process. Comparison of the average relative translations (a) and the relative change of angle of gripper 4 (b)

\section{Geometry 2}

As mentioned above, the simulation setup remains unchanged for geometry 2. Besides a biaxial layup, also a quasi-isotropic layup is investigated. It becomes apparent, that a defect-free forming is possible for the biaxial layup (cf. Figure 6(c)), whereas distinct wrinkling behavior is observed for the quasi-isotropic layup (cf. Figure 6(a)). To investigate wrinkling behavior, forming results for a remaining tool stroke of approximately $5 \mathrm{~mm}$ are presented for the quasi-isotropic layup instead of the results for the fully closed mold.

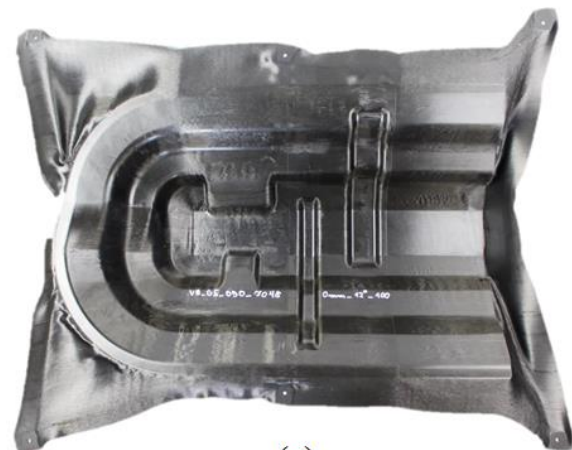

(a)

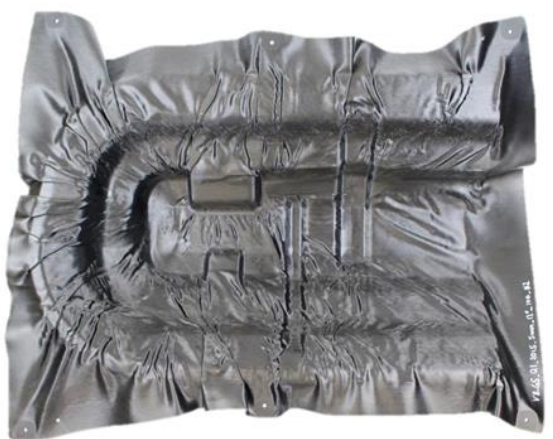

(c)

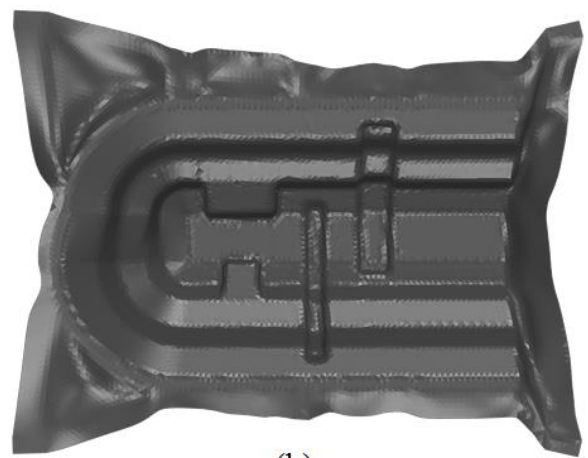

(b)

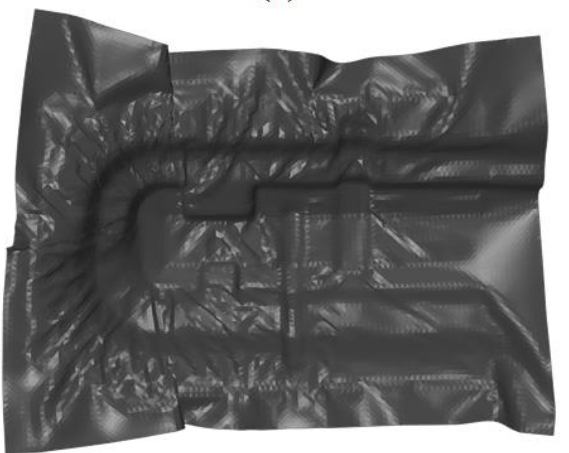

(d)

FIGURE 6. Comparison of experimental results $(a, c)$ to simulation results $(b, d)$ for a biaxial layup and fully close mold (a,b) and a quasi-isotropic layup and remaining tool stroke of $5 \mathrm{~mm}(\mathrm{c}, \mathrm{d})$, respectively. 
Comparing the forming simulation results of the biaxial layup (cf. Figure 6(d)) and the quasi-isotropic layup (cf. Figure 6(b)) to the according experimental tests, a good agreement is observed for the outer contour. Beyond that, also wrinkling behavior is predicted in very good agreement to the experimental tests for the quasi-isotropic layup.

\section{DISCUSSION AND CONCLUSION}

An advanced gripper system for online monitoring of gripper forces, translations and rotations during thermoforming is presented. Beyond that, modeling techniques for gripper-assisted FE forming simulation are suggested and validated by means of application to a complexly shaped, generic geometry and the comparison of the forming simulation results to experimental tests.

The presented enhanced gripper system allows a reliable process monitoring of gripper forces, translations and rotations. The measurements provide crucial data for FE forming simulation, as membrane forces are usually mostly unknown. Once the applied forces are known, FE forming simulation is capable of reliable predictions of forming behavior, as shown in this study by the identification, parametrization and validation of boundary conditions on geometry 1 and the transfer of the developed modelling approaches to geometry 2 . Furthermore, the comparison of translations and rotation of the grippers, while modeling gripper kinematics, shows a good agreement to experimental tests for geometry 1 and a good prediction of wrinkling behavior for geometry 2.

Based on the presented results and modeling techniques for gripper-assisted forming, the application of the modeling approaches is enabled for a reliable process optimization. Consequently, the optimal number and position of grippers, as well as required force magnitudes and directions are predictable, preventing a time and cost expensive "trial and error" process design.

\section{ACKNOWLEDGEMENT}

The authors would like to thank the German Federal Ministry of Education and Research for the funding of the

project SMiLE (03X3041P), for which the presented work is carried out, as well as MoPaHyb in which the developments in online process monitoring during forming were performed.

\section{REFENENCES}

1. E. Fuchs, F. Field, R. Roth, R. Kirchain, Strategic materials selection 365 in the automobile body: Economic opportunities for polymer composite design, Composites Science and Technology 68 (9) (2008) 1989-2002.

2. J. Verrey, M. D. Wakeman, V. Michaud, J.-A. Månson, Manufacturing cost comparison of thermoplastic and thermoset rtm for an automotive 21370 floor pan, Composites Part A: Applied Science and Manufacturing 37 (1) (2006) 9-22.

3. T. Joppich, D. Doerr, L. van der Meulen, T. Link, B. Hangs and F. Henning, "Layup and Process Dependent Wrinkling Behavior of PPS/CF UD Tape-Laminates during Non-Isothermal Press Forming Into a Complex Component" Proceedings ESAFORM 2016 Conference, Nantes, 2016.

4. D. Dörr, F. Schirmaier, F. Henning and L. Kärger, A viscoelastic approach for modeling bending behavior in Finite Element forming simulation of continuously fiber reinforced composites, Composites: Part A, 2016, in press, doi: http://dx.doi.org/10.1016/j.compositesa.2016.11.027.

5. D. Dörr, T. Joppich, F. Schirmaier, T. Mosthaf, L. Kärger and F. Henning, A Method for Validation of Finite Element Forming Simulation On Basis of a Pointwise Comparison of Distance and Curvature, Proceedings ESAFORM16, Nantes (France), 2016.

6. Dassault Systemes (ed.), Abaqus Analysis User's Guide, Abaqus Documentation 6.14, 2015 